\title{
Epidemiología de la Difteria en el niño, área de Valdivia 1975 - 1978
}

Dr. J. Jars S.* Dr. R. Burgos L.** Srta. M. Mediavilla***

\begin{abstract}
Epidemiologic aspect of diphteria in Valdivia pediatric population in the period 1975 - 1978
\end{abstract}

\begin{abstract}
A statistical review of D.P.T. and D.T. inmunization was done, annalizing the covering for both in the period between 1975 - 1978 .

The case repport of Diphteria in the population under 15 years and the clinical aspects of 67 cases, admitted to the infectous disease Unit, Pediatric Service, Regional Hospital Valdivia, were studied during the same period.

A satisfactory coveting of immunization for the first dose of D.P.T. was observed, with a progresive decrease in the next following doses.

In spite of a good percentage of DT covering, School age children showed the highest index of morbility.

It is speculated about the posible explanation of that finding. We make enphasis in the importance of complete D.P.T. vaccinal series in order to get specifical protective antibodies and change the current epidemiological situation.
\end{abstract}

Se describe clásicamente a la difteria como una enfermedad infecto contagiosa aguda altamente difusible que se mantiene en forma endémica presentando brotes en las estaciones frías del año. frías del año.

El agente etiológico es el Corinebacterium Difteria, cuyo poder patógeno está deterninado por la reacción inflamatoria local (formación de pseudomembrana) y por una potente exotoxina que compromete diferentes parénquimas vitales.

\section{*Unidad de Infecciosos Servicio Pediatria Hospital Regional} Valdivia

- Unidad de Infecciosos. Instituto de Pediatris UACh.

***Interma de Medicina, Escuela de Medicina, UACh.
La localización más frecuente es la respiratoria faucial y afecta principalmente a preescolares y escolares. Se cuenta actualmente en nuestro medio con elementos preventivos y terapéuticos destinados a erradicar esta enfermedad; sin embargo, aún existen áreas con alta tasa de morbimortalidad. En Chile durante 1977 la morbilidad fue de un 5.2. $x$ 100.000 habitantes y la mortalidad, $0,5 \times$ 100.000 .

En el Area de Valdivia, la morbilidad fue de 19.4 por 100.000 y la mortalidad alcanzó un 2,3 por 100.000 , siendo para ese año la cifra más alta del país.

La observación sistemática de casos de Difteria, clínica y bacteriologicamente comprobados en el 
Area de Salud Valdivia entre los años 1975 - 1978, nos ha motivado a revisar el problema desde el punto de vista epidemiológico a fin de poder establecer algunas variables que lleven a que la situación en nuestra área no sea la clásicamente conocida.

\section{MATERIAL Y METODO}

El presente estudio se realizo en el Servicio de
Pediatria Hospital Regional Valdivia, y comprende la revisión estadistica de inmunizaciones con vacuna triple y toxoide diftérico, las denuncias de la enfermedad en la población menor de 15 años en el Area de Salud Valdivia entre los años 1975 1978, y el análisis de 67 casos de Difteria que ingresaron a la Unidad de Infecciosos durante dicho período.

RESULTADOS: (Grafico N..$^{\circ}$ )

\section{Gráfico 1}

GRADO DE CUMPLIMIENTO DEL PROGRAMA DE INMUNIZACION CON VACUNA TRIPLE, POR DOSIS EN EL AREA DE LA SALUD VALDIVIA AÑOS $1975-1978$.

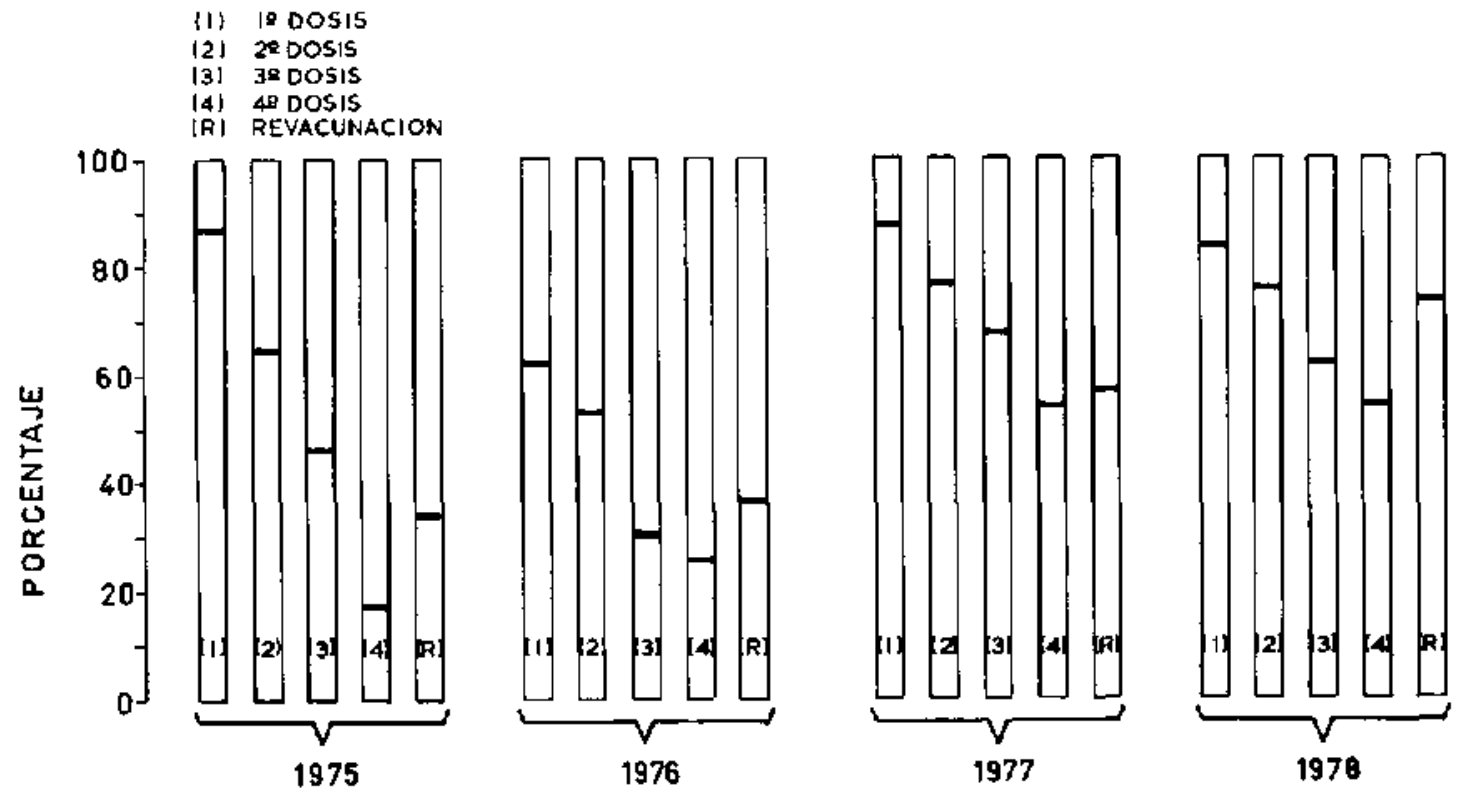

Grífico 2 Grado de cumplimiento del programa de inmunización con toxoide Diftérico en el Area de Salud Valdivia anos $1975-1978$
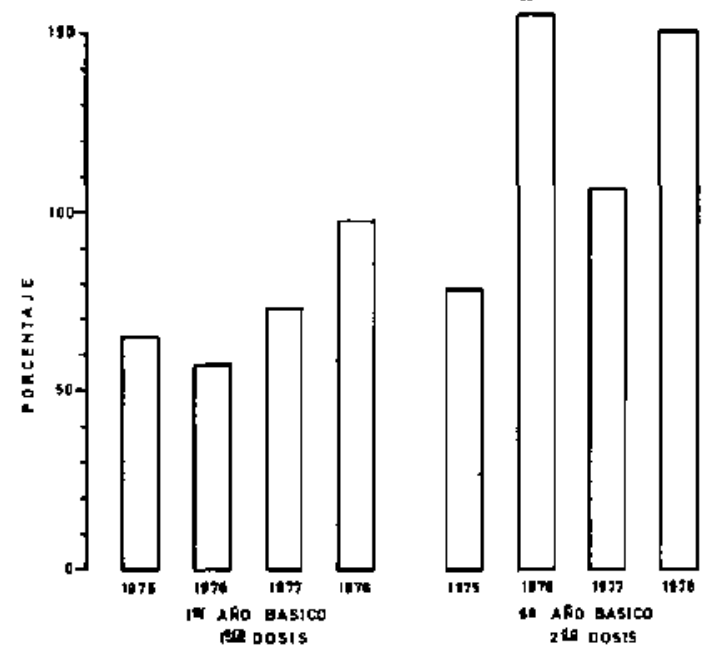

Se puede comprobar que para la primera dosis de DPT se logró un curnplimiento que en los años considerados oscila entre un 62 y $87.5 \%$. Estas cifras se reducen en forma progresiva y ostensible en las siguientes dosis de la serie primaria de inmunización, por ejemplo para la cuarta dosis el grado de cumplimiento varia entre un 17.8 y $54.1 \%$, no obstante se evidencia un incremento del rendimiento para las distintas dosis que oscila entre un 10 y $35 \%$ aproximadamente para el año 1978 en relación al año de referencia 1975.

Sin embargo las inmunizaciones con toxoide diftérico mantienen un rendimiento ascendente de 64,9 a $97,9 \%$ para la primera dosis, en cambio para la segunda se observan cifras de cumplimiento que sobrepasan al $150 \%$ de lo programado, lo que pudiera significar que la programación 
no fue en relación a la población real del área. Además se incluyen dosis administradas en canpañas extraordinarias realizadas en 1977 y 1978 , y las dosis administradas a los contactos directos de casos de difteria clínica denunciados en dicho periodo.

Tabla 1

Morbilidad por Diftéria expresada en tasas por 100.000 habitantes en población menor de 15 años Area de Valdivia años $1975-1978$

\begin{tabular}{cccc}
\hline Año & N. ${ }^{\circ}$ Denuncias & Población & Tasa 100.000 Hsb. \\
\hline 1975 & 28 & 116.062 & 2,4 \\
1976 & 44 & 115.662 & 3,8 \\
1977 & 39 & 118.229 & 3,3 \\
1978 & 55 & 115.664 & 4,7 \\
\hline
\end{tabular}

Ref: Servicio Estadistica Hospital Regional Valdivia.

Al estudiar el N..$^{\circ}$ de denuncias de la enfermedad tomada en tasas por 100.000 habitantes, se observa un incremento de la morbilidad a casi un
100\% para 1978 en relación al año de referencia 1975 , mientras quic + población no muestra variación numérica significativa.

Tabla 2

Mortalidad por Difteria, expresada en tasas por 100.000 habitantes en poblacion menores de 15 años, Area de Valdivia año 1975 - 1978.

\begin{tabular}{cccc}
\hline Año & N..$^{\circ}$ Muertos & Población & Tasa por 100.000 Hab. \\
\hline 1975 & 2 & 116.062 & 1,7 \\
1976 & 2 & 115.662 & 1,7 \\
1977 & 7 & 118.229 & 5,9 \\
1978 & 5 & 115.669 & 4,3 \\
\hline
\end{tabular}

Las tasas de mortalidad también muestran un incremento progresivo y para el año 1978 alcanza a más de un $260 \%$ en relación a 1975 . Estas tasas de mortalidad constituyen aproximadamente un $10 \%$ de las de morbilidad antes analizadas.

Tabla 3

Procedencia de 166 denuncias por Difteria en menores de 15 años según localidad Area de Valdivia años $1975-1978$.

\begin{tabular}{|c|c|c|c|c|c|c|c|c|}
\hline \multirow[t]{2}{*}{ Localidad } & \multicolumn{2}{|c|}{1975} & \multicolumn{2}{|c|}{1976} & \multicolumn{2}{|c|}{1977} & \multicolumn{2}{|c|}{1978} \\
\hline & N. ${ }^{\circ}$ & $\%$ & $\mathrm{~N}^{\circ}{ }^{\circ}$ & $\%$ & N. ${ }^{\prime}$ & $\%$ & N. ${ }^{\circ}$ & $\%$ \\
\hline Paillaco & 2 & 7 & 6 & 13 & 9 & 23 & 30 & 54 \\
\hline Valdivia & 19 & 67 & 6 & 13 & 7 & 17 & 7 & 12 \\
\hline Río Buenu & 0 & 0 & $\mathbf{5}$ & 11 & 1 & 2 & 10 & 18 \\
\hline Panguipulli & 2 & 7 & 7 & 15 & 5 & 12 & 1 & 2 \\
\hline Lad Unión & 0 & 0 & 12 & 27 & 14 & 35 & 3 & 5 \\
\hline Lanco & 3 & 10 & 1 & 2 & 0 & 0 & 1 & 2 \\
\hline Los Lagos & 2 & 7 & 3 & 6 & 0 & 0 & 2 & 3 \\
\hline San José & 0 & 0 & 4 & 9 & 2 & 5 & 1 & 2 \\
\hline Corral & 0 & 0 & 0 & 0 & 1 & 2 & 0 & 0 \\
\hline Total & 28 & 100 & 44 & 100 & 39 & 100 & 55 & 100 \\
\hline
\end{tabular}

Hef: Servicio Eistadístiço Hospital Regional Valdivia 
En relación a la procedencia, se observa que más de un 50\% para el año 1978 procede de Paillaco, pudiêndose además comprobar en esta tabla un aumento sostenido de la incidencia anual.

No hay diferencias significativas desde el punto de vista numérico entre procedencia urbana y rural, pero dado que para el área dë Valdivia se tiene un $57 \%$ de población urbana, significaria que hay una mayor incidencia en población nural.

En relación a la distribución etaria se evidencia un franco predorninio para todos los años analizados del grupo etario de 5 a 9 (41 a $52 \%)$.

En relación a la frecuencia mensual para 1978 , se encontró un incremento notable en los meses de agosto y septiembre, en relación al promedio entre los años $1975-1977$, lo que traduce un claro brote epidémico.
Gráfico 3

Procedencia en 166 casos de Difteria en menores de 15 años Area de Valdivia años 1975 - 1978.

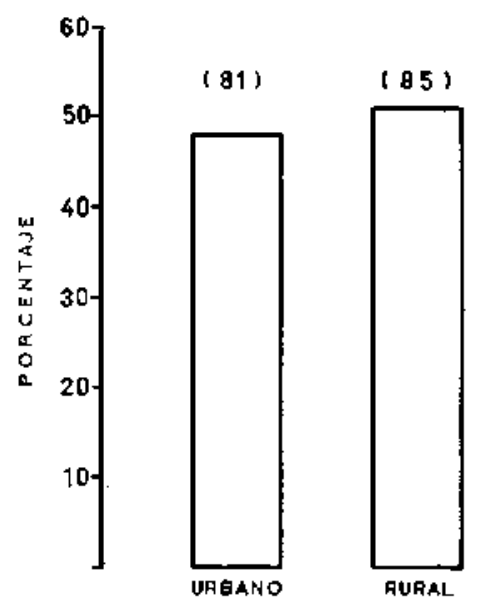

Tabla 4

Distribución etaria en 166 denuncias por Difteria en menores de 15 años, Area de Salud Valdivia Años 1975 - 1978.

\begin{tabular}{lrlllllll}
\hline Grupo Etario & 1975 & $\%$ & 1976 & $\%$ & 1977 & $\%$ & 1978 & $\%$ \\
\hline $0-4$ años & 6 & 21.4 & 13 & 29.5 & 9 & 23 & 9 & 16.3 \\
$5-9$ años & 14 & 50 & 19 & 43.1 & 16 & 41 & 29 & 52.7 \\
$10-14$ años & 8 & 28.5 & 12 & 27.2 & 14 & 35.8 & 17 & 30.9 \\
\hline Total & 28 & 100 & 44 & 100 & 39 & 100 & 55 & 100 \\
\hline
\end{tabular}

Feferencia: Servicio Estadistica Hospital Regional Valdivia.

\section{Gráfico 4}

Distribución mensual de 166 denuncias por Difteria en menores de 15 añus Area de Salud Valdivia Años $1975-1978$.

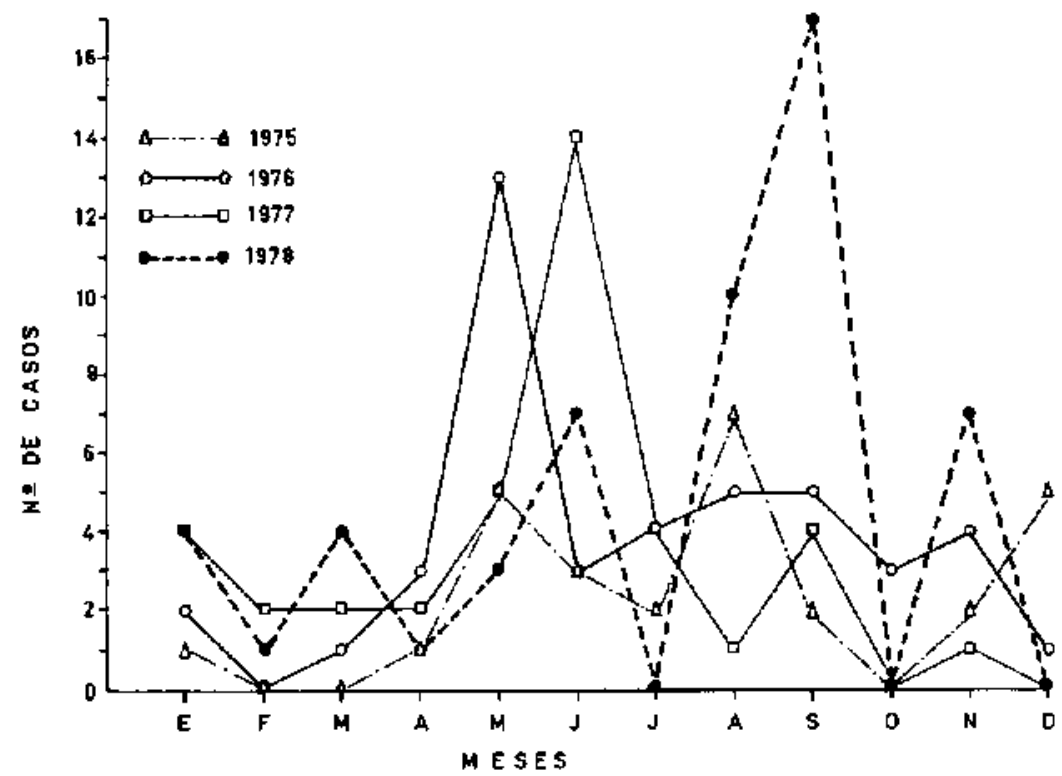

Ra SERV ESTADISTICA HOSPITAL REGIONAL VALDIVIA. 
Durante este período ingresaron a la Unidad de Infecciosos, Servicio de Pediatría Valdivia, 67 casos de Difteria.

Tabla 5

Clasificación de 67 casos de Difteria Servicio Pediatria Unidad Infecciosos Valdivia 1975 - 1978.

\begin{tabular}{lcc}
\hline Tipo & N. ${ }^{\circ}$ Casos & Porcentaje \\
\hline Común & 12 & 18 \\
Submaligna & 41 & 61 \\
Maligna & 14 & 21 \\
\hline Total & 67 & 100.
\end{tabular}

Desde el punto de vista clínico, el mayor porcentaje corresponde a la forma faucial submaligna (61\%) de acuerdo a la clasificación de Marfán, lo que no concuerda con lo habitualmente descrito que asigna mayor frecuencia al tipo común, esto pudiera explicarse por la no referencia de estos casos desde los Hospitales periféricos, por no constituir gravedad manifiesta y/o al diagnóstico incorrecto de este tipo de angina (etiolágico).

\section{Gráfico 5}

Procedencia en 67 casos de Difteria, Servicio de Pediatria. Hospital Regional Valdivia Unidad Infecciosos Años $1975 \cdot 1978$.

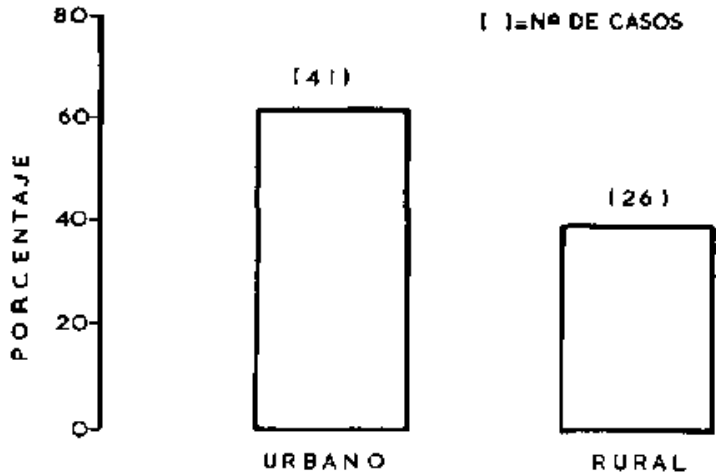

Con respecto a la procedencia, encontramos un $61 \%$ de pacientes urbanos.

\section{Tabla 6}

Distribución etaria en 67 cusos Difteria Servicio de Pediatría Unidad Infecciosos Valdivia Años 1975 - 1978.

\begin{tabular}{lcl}
\hline Grupo etario & N. de casos & $\%$ \\
\hline $0-4$ años & 1,4 & 20.8 \\
$5-9$ años & 3,3 & 49.3 \\
$10-14$ años & 2,0 & 29.9 \\
\hline
\end{tabular}

En cuanto a la distribución etaria, corresponde también una frecuencia de prácticamente un $50 \%$ para el grupo de 5 a 9 años.

\section{Gráfico 6}

Distribución por sexo en 67 casos Difteria, Servicio de Pediatria Unidad Infecciosos Valdivia Años 1975 - 1978.

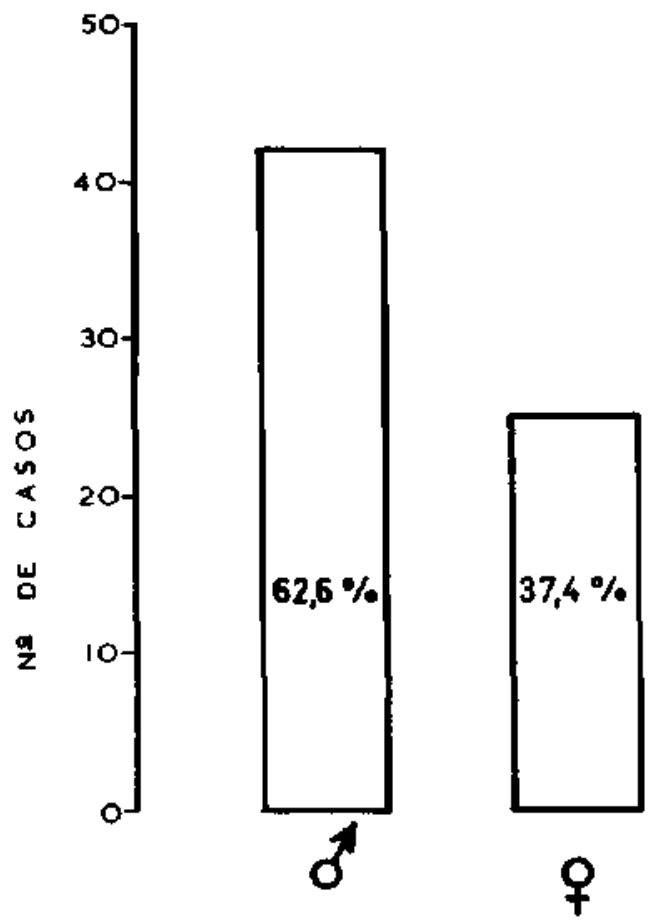

En cuanto a distribución por sexo, obtuvimos un $62 \%$ masculino, para lo que no tenemos explicación clara.

\section{Cräfico 7}

Distribución estacional de 67 casos de Difteria Servicio de Pediatria Unidad de Infecciosos Años 1975 - 1978 .

$$
\text { 1) N*CASOS }
$$

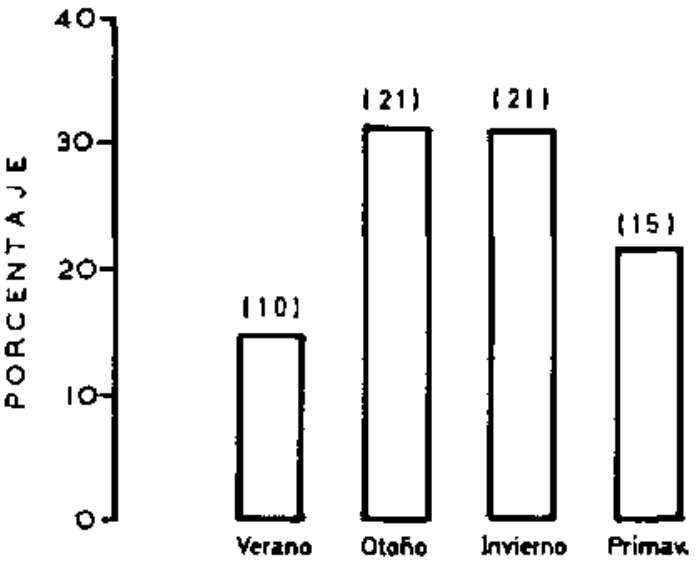


En relación a la distribución estacional, lo encontrado concuerda con lo clásicamente descrito en el sentido de una mayor frecuencia en otoño $e$ invierno.

\section{Gráfico 8}

Antecedentes de inınunización con vacuna triple y/o Toxoide Diftérico en 67 casos de Difteria Servicio de Pediatria Unidad Infecciosos, Valdivia Años 1975 - 1978.

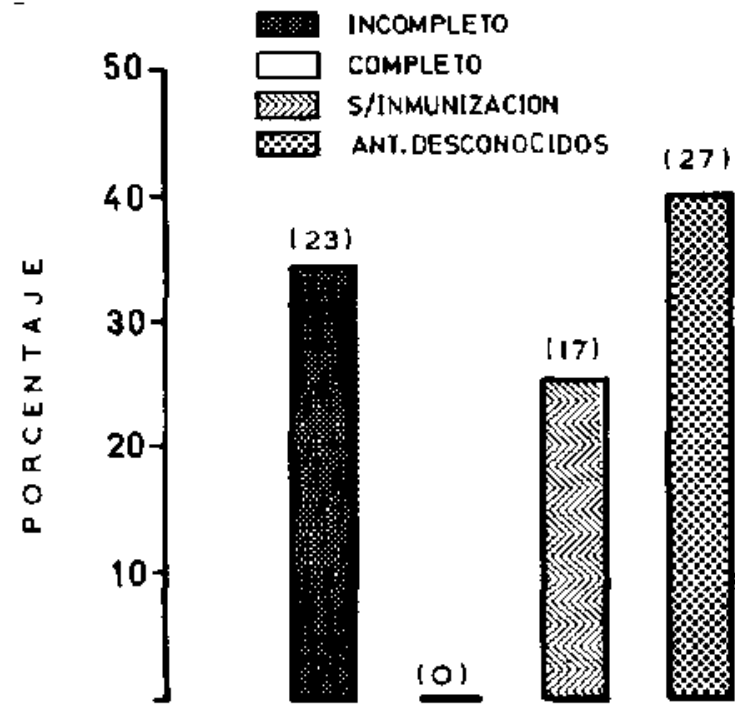

Respecto al grado de inmunización observamos una mayor frecuencia de antecedentes desconocidos, incompletos, y carentes en lo absoluto de vacunas, sin que se registrara ningún caso con la serie de inmunización completa, lo que concuerda con lo más frecuentemente reportado.

Tabla 8

Poncentaje de inuertes según tipo clínico en 67 casos Difteria Servicio de Pediatría, Lnidad infecciosos Años 1975 - 1978.

\begin{tabular}{lccc}
\hline Tipo clínico & $\begin{array}{c}\text { N. cle } \\
\text { casos }\end{array}$ & $\begin{array}{c}\text { N." de } \\
\text { muertes }\end{array}$ & Porcentaje \\
\hline Común & 1,2 & 0 & 0 \\
Submaligno & 4,1 & 3 & 7,3 \\
Maligno & 1,4 & 8 & 57,1 \\
\hline Total & 67 & 11 & 16,4
\end{tabular}

Referente al porcentaje de muerte según tipo clínico, se encontró un $\mathbf{5 7 \%}$ para el tipo maligno, no observándose mortalidad en el tipo común, lo que también concuerda con lo descrito habitual- mente. La mortalidad global en nuestra serie durante los años analizados fue de un $16.4 \%$.

\section{COMENTARIO}

Pese a ser la Difteria una enfermedad infectocontagiosa susceptible de erradicar con los métodos actualmente en uso (vacuna, antitoxina, antibióticos) se mantiene como uno de los más agudos problemas de salud pública en nuestra área.

Del análisis de esta revisión se concluye:

1) No existe un incremento significativo de la población más susceptible (menores de 15 años) con respecto a año de referencia 1975.

2) Las denuncias por difteria muestran un aumento sostenido en el periodo que se analizó, lo cual por una parte señala la alta incidencia de la enfermedad en el área y por otra pudiera significar un adecuado y oportuno tratamiento de los casos indices como también de los contactos del paciente diftérico.

3) Las condiciones socioeconómicas y ambientales de esta área dentro de las cuales deben destacarse condiciones de nacimiento, climáticas, alta ruralidad, bajo nivel cultural, y una falta de conciencia respecto al problema por nuestra comunidad, pudiera motivar que la difusibilidad de la infección aumente.

4) Para los años estudiados se consideran aceptables las coberturas con la primera dosis de vacuna triple, no así con las siguientes dosis de la serie primaria.

5) Resulta paradójico que mientras alcanza un mayor grado de cumplimiento en las dosis de refuerzo con toxoide en el grupo de escolares, éstos muestran una tasa específica de morbilidad mayor.

Esto se explica considerando que un gran porcentaje de la población escolar no recibió o sólo se le administró parcialmente la serie primaria de vacunación con DPT., lo cual traduce una protección parcial o inexistente durante los primeros años de vida del niño.

\section{REFERENCIAS}

\footnotetext{
${ }^{1}$ Robbins Stanley L. Tratado de Patologia. 3a Edicion, 1968.

2Vaushan Nelson ac Kay. Tratado de Pediatria (Vol. II). 6. Edición, 1971.

${ }^{3}$ Menegheflo, Juliu. Pediatra 2a . Edición. 1978.

${ }^{4}$ Zaror L., Otth L., Fernúndez H., Microbiologia Sistemática y Clinica. UACh, 1976.

5 Enfermedades de notificación obligatoria. Anuario 1977, Ministerio de Salud, Departamento de Planificación.

6 Armijo Rojas R. Epidemiologir (Vol. JI). J ${ }^{3}$. Edicion, 1976.
} 